\title{
An Analysis of Multi-Sampled Issue and No-Replacement Tournament Selection
}

\author{
Huayang Xie \\ Mengjie Zhang \\ Peter Andreae \\ Victoria University of Wellington \\ Wellington, New Zealand \\ \{hxie,mengjie,pondy,markj\}@mcs.vuw.ac.nz \\ Mark Johnston
}

\begin{abstract}
Standard tournament selection samples individuals with replacement. The sampling-with-replacement strategy has its advantages but also has issues. One of the commonly recognised issues is that it is possible to have the same individual sampled multiple times in a tournament. Although the impact of this multi-sampled issue on genetic programming is not clear, some researchers believe that it may lower the probability of some good individuals being sampled or selected. One solution is to use an alternative tournament selection (no-replacement tournament selection), which samples individuals in a tournament without replacement. This paper analyses no-replacement tournament selection to investigate the impact of the scheme and the importance of the issue. Theoretical simulations show that when common tournament sizes and population sizes are used, noreplacement tournament selection does not make the selection behaviour significantly different from that in the standard one and that the multi-sampled issue seldom occurs. In general, the issue is not crucial to the selection behaviour of standard tournament selection.
\end{abstract}

\section{Categories and Subject Descriptors}

I.6 [Simulation and Modelling]: Model Validation and Analysis

\section{General Terms}

Theory

\section{Keywords}

Tournament Selection, Multi-Sampled Issue, Modelling, Simulation

\section{INTRODUCTION}

Tournament selection is one of the commonly used parent selection schemes in Evolutionary Algorithms (EAs) and is

Permission to make digital or hard copies of all or part of this work for personal or classroom use is granted without fee provided that copies are not made or distributed for profit or commercial advantage and that copies bear this notice and the full citation on the first page. To copy otherwise, to republish, to post on servers or to redistribute to lists, requires prior specific permission and/or a fee.

GECCO'08, July 12-16, 2008, Atlanta, Georgia, USA.

Copyright 2008 ACM 978-1-60558-130-9/08/07 ...\$5.00. very popular in Genetic Programming (GP) [10]. According to the description given by Goldberg and Deb [8], the initial study of tournament selection can be traced back to the early 1980s [5]. One form of the conventional tournament selections introduced in [5] has became the standard. Standard tournament selection randomly samples $k$ individuals with replacement from the current population of size $N$ into a tournament of size $k$ and selects the one with the best fitness from the tournament. Because individuals in a tournament are sampled from the population with replacement, standard tournament selection is simple to code and efficient for both non-parallel and parallel architectures [14]. Standard tournament selection has been widely studied theoretically since the 1990 s $[1,2,3,14,17]$, while many alternative implementations have been developed $[7,9,11,12$, 19].

One commonly recognised issue in standard tournament selection is that it is possible to have the same individual sampled multiple times in a single tournament (the multisampled issue). Although the impact of this multi-sampled issue on GP is not clear, some researchers believe that it may lower the probability of some good individuals being sampled or selected. One solution is to use an alternative tournament selection in which individuals are sampled without replacement into a tournament, then, after the winner is determined, all individuals in the tournament are returned to the population. According to [8], tournament selection using the sampling-without-replacement strategy is the other conventional tournament selection. We abbreviate it as noreplacement tournament selection. It is not clear why noreplacement tournament selection is less commonly used in EAs.

From the literature, it is not clear whether the samplingwithout-replacement strategy makes the selection behaviour significantly different from that in the standard one and whether the multi-sampled issue is crucial to the selection behaviour in standard tournament selection. Although researchers may have an intuition that the two methods are not significantly different, this view has not yet been well proved.

\subsection{Goals}

This paper theoretically analyses no-replacement tournament selection to investigate the following questions:

- Does the selection behaviour in no-replacement tournament selection differ significantly from that in standard tournament selection?

- Does the multi-sampled issue significantly influence 
the selection behaviour in standard tournament selection?

In order to answer these questions, we need to model noreplacement tournament selection, measure its selection behaviour, and compare it with the standard one.

\section{BACKGROUND}

\subsection{Measurement of Selection Pressure in Tournament Selection}

Selection pressure is defined as the degree to which the better individuals are favoured [13]. It gives individuals of higher quality a higher probability of being used to create the next generation so that EAs can focus on promising regions in the search space [2].

In tournament selection, the mating pool consists of tournament winners. It has a higher average fitness than the average population fitness. The fitness difference reflects the selection pressure, which is expected to improve the fitness of each succeeding generation [13].

There are several measurements for selection pressure in different contexts, including takeover time, selection intensity, loss of diversity, reproduction rate, and selection probability distribution.

Takeover time is introduced by Goldberg and Deb [8] to quantify the selection pressure. It is defined as the number of generations required to completely fill a population with just copies of the best individual in the initial generation when only selection and copy operators are used. The larger the takeover time, the lower the selection pressure. Goldberg and Deb estimated the takeover time for standard tournament selection using the asymptotic expression

$$
\frac{1}{\ln k}(\ln N+\ln (\ln N))
$$

where $N$ is the population size and $k$ is the tournament size. The approximation improves as $N \rightarrow \infty$. However, this measure is static and constrained and therefore does not reflect the selection behaviour dynamics from generation to generation in EAs.

Selection intensity is another measure for selection pressure. It was first introduced in the context of population genetics to obtain a normalised and dimensionless measure [6], and, later was adopted and applied to GAs [16]. Blickle and Thiele $[2,3]$ used it in the same way as Bulmer [6]. They measured selection intensity using the expected change of the average fitness of the population. As the measurement is dependent on the fitness distribution in the initial generation, they assumed the fitness distribution followed the normalised Gaussian distribution and introduced an integral equation for modelling selection intensity in standard tournament selection. For their model, analytical evaluation can be done only for smaller tournament sizes and numerical integration is needed for larger tournament size. Also the model is not valid in the case of discrete fitness distributions. Another limitation is the assumption of the fitness distribution followed the normalised Gaussian distribution, which is not valid in general [18]. Further, because the actual fitness values are ignored and only relative rankings are used in tournament selection, the model is of limited use.

Loss of diversity is defined as the proportion of individuals in a population that are not selected during the selection phase $[2,3]$. Blickle and Thiele estimated the loss of diversity in standard tournament selection as:

$$
k^{-\frac{1}{k-1}}-k^{-\frac{k}{k-1}}
$$

However, Motoki [15] pointed out that Blickle and Thiele's estimation of the loss of diversity in tournament selection does not follow their definition, and indeed Blickle and Thiele's estimation is of loss of fitness diversity. Motoki recalculated the loss of program diversity in a wholly diverse population, i.e., every individual has distinct fitness value, on the assumption that the worst individual is ranked 1st, as:

$$
\frac{1}{N} \sum_{j=1}^{N}\left(1-\frac{j^{k}-(j-1)^{k}}{N^{k}}\right)^{N}
$$

where $j$ is the individual rank and $\left(1-\frac{j^{k}-(j-1)^{k}}{N^{k}}\right)^{N}$ is the probability that the individual has never been selected in $N$ tournaments.

Reproduction rate is defined as the ratio of the number of individuals with a certain fitness $f$ after and before selection $[2,3]$. A reasonable selection method should favour good individuals by giving them a high ratio and punish bad individuals by giving a low ratio. Branke et al. [4] introduced a similar concept which is the expected number of copies of an individual selected in the selection phase. It is calculated by the selection probability of the individual in a single tournament multiplied by the total number of tournaments. The concept is termed selection frequency in this study instead of using reproduction rate that has another meaning in GP. They provided a model to calculate the selection frequency for an individual of rank $j$ in standard tournament selection in a wholly diverse population on the assumption that the worst individual is ranked 1st, as:

$$
N \frac{j^{k}-(j-1)^{k}}{N^{k}}
$$

Selection probability distribution of a population is defined to consist of the probabilities of each individual in the population being selected at least once in the selection phase [20]. It is illustrated in [20] as a three dimensional graph, providing a thorough picture of the selection behaviour over the population during the whole selection phase.

\subsection{Models of Sampling Behaviour and Selec- tion Behaviour in Tournament Selection}

There are a number of papers modelling and comparing the selection behaviour of a variety of selection schemes $[1$, $3,4,8,14,15]$. Dedicated studies on standard tournament selection include $[2,13,17,20]$.

Based on the concept of takeover time [8], Bäck [1] compared several selection schemes, including tournament selection. He presented the selection probability of an individual of rank $j$ in one tournament for a minimisation $\operatorname{task}^{1}$, with an implicit assumption that the population is wholly diverse, as:

$$
N^{-k}\left((N-j+1)^{k}-(N-j)^{k}\right)
$$

In order to model the expected fitness distribution after performing tournament selection in a population with a more general form, Blickle and Thiele [2] extended the selection probability model in [1] to describe the selection

\footnotetext{
${ }^{1}$ Therefore the best individual is ranked 1 st.
} 

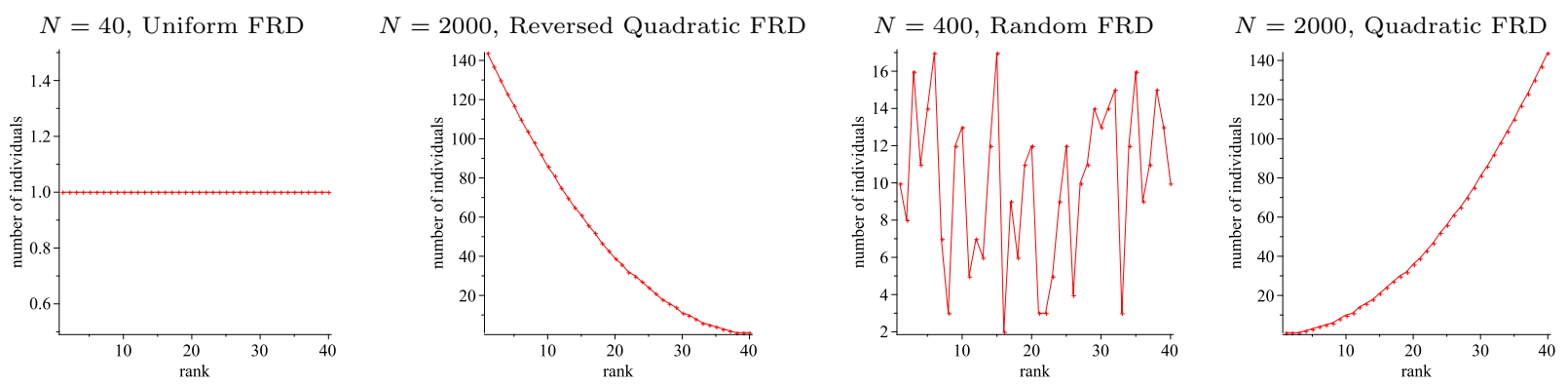

Figure 1: Four populations with different fitness rank distributions.

probability of individuals with the same fitness. The model is quite abstract although it is quite elegant. Their model ranks the worst individual 1st, and introduces the cumulative fitness distribution, $S\left(f_{j}\right)$, which denotes the number of individuals with fitness value $f_{j}$ or worse. They showed the selection probability of individuals with rank $j$ as:

$$
\left(\frac{S\left(f_{j}\right)}{N}\right)^{k}-\left(\frac{S\left(f_{j-1}\right)}{N}\right)^{k}
$$

In order to demonstrate the computational savings in the Backward-chaining Evolutionary Algorithms, Poli and Langdon [17] calculated the probability that one individual is not sampled in one tournament as $1-\frac{1}{N}$, then consequently the expected number of individuals not sampled in any tournament as:

$$
N\left(\frac{N}{N-1}\right)^{-k y}
$$

where $y$ is the total number of tournaments required to form an entire new generation.

In order to illustrate that selection pressure is insensitive to population size in standard tournament selection in a population with a more general form, we [20] presented a sampling probability model that any program $p$ is sampled at least once in $y \in\{1, \ldots, N\}$ tournaments as:

$$
1-\left(\left(\frac{N-1}{N}\right)^{N}\right)^{\frac{y}{N} k}
$$

and a selection probability model that a program $p$ of rank $j$ is selected at least once in $y \in\{1, \ldots, N\}$ tournaments as:

$$
1-\left(1-\frac{\left(\frac{\sum_{i=1}^{j}\left|S_{i}\right|}{N}\right)^{k}-\left(\frac{\sum_{i=1}^{j-1}\left|S_{i}\right|}{N}\right)^{k}}{\left|S_{j}\right|}\right)^{y}
$$

where $\left|S_{j}\right|$ is the number of programs of the same rank $j$.

\section{ASSUMPTIONS AND DEFINITIONS}

In general, a population can be partitioned into bags consisting of programs with equal fitness. These "fitness bags" may have different sizes. As each fitness bag is associated with a distinct fitness rank, we can characterise a population by the number of distinct fitness ranks and the size of each corresponding fitness bag, which we term fitness rank distribution (FRD). If $S$ is the population, then we use the notation $N$ to be the size of the population, $S_{j}$ to be the bag of programs with the fitness rank $j$ and $\left|S_{j}\right|$ to be its size. We denote tournament size by $k$ and rank the program with the worst fitness 1st. We follow the standard breeding process, that is, one parent produces one offspring after mutation and two parents produce two offspring via crossover. Therefore $N$ tournaments are required to generate all individuals in the next generation.

To theoretically analyse the selection behaviour, this paper uses the loss of program diversity [15], the selection frequency [4], and the selection probability distribution [20] on four populations with different FRDs: uniform, reversed quadratic, random, and quadratic.

The four FRDs are designed to simulate the four stages of evolution. The uniform FRD represents the initialisation stage, where each fitness bag has an equal number of programs. A typical case of the uniform fitness rank distribution can be found in a wholly diverse population. The reversed quadratic FRD represents the early evolving stage, where commonly only a small number of individuals have better fitness values. The random FRD represents the middle stage of evolution, where better and worse individuals are possibly randomly distributed. The quadratic FRD represents the later stage of evolution, where a large number of individuals converge to better fitness values.

Since the impact of population size on selection behaviour is unclear, we tested several different commonly used population sizes, ranging from small to large. Due to space constraints and for reporting convenience, we only illustrate the results for three population sizes, namely 40, 400, and 2000, for the uniform FRD, the random FRD, and the reversed quadratic and quadratic FRDs respectively. Note that although the populations with different FRDs are of different sizes, the number of distinct fitness ranks is designed to be the same value 40 for easy visualisation and comparison purposes (see Figure 1).

We also studied other different numbers of distinct fitness ranks, including 100, 500 and 1000. Since the results were consistent, we omitted them due to space constraints.

\section{MODELLING NO-REPLACEMENT TOUR- NAMENT SELECTION}

This section describes the probabilities that a program is first sampled, and then selected in a series of tournaments.

The only factor making no-replacement tournament selection different from the standard one is that any individual in a population will be sampled at most once in a single tournament. Therefore, let $D$ be the event that an arbitrary program $p$ is drawn or sampled in a tournament of size $k$. The probability of the event $D$ is: 


$$
P(D)=\frac{k}{N}
$$

The probability of the event $I_{y}$ that $p$ is drawn or sampled at least once in $y \in\{1, \ldots, N\}$ tournaments is:

$$
\begin{aligned}
P\left(I_{y}\right) & =1-(1-P(D))^{y} \\
& =1-\left(1-\frac{k}{N}\right)^{y} \\
& =1-\left(\left(\frac{N-k}{N}\right)^{N}\right)^{\frac{y}{N}}
\end{aligned}
$$

Lemma 1. For a particular program $p \in S_{j}$, the probability of the event $E_{j, y}$ that $p$ is selected at least once in $y \in\{1, \ldots, N\}$ tournaments is:

$$
P\left(E_{j, y}\right)=1-\left(1-\frac{\frac{\left(\begin{array}{c}
\sum_{i=1}^{j}\left|S_{i}\right| \\
k
\end{array}\right)}{\left(\begin{array}{c}
N \\
k
\end{array}\right)}-\frac{\left(\begin{array}{c}
\sum_{i=1}^{j-1}\left|S_{i}\right| \\
k
\end{array}\right)}{\left(\begin{array}{c}
N \\
k
\end{array}\right)}}{\left|S_{j}\right|}\right)^{y}
$$

Proof. The probability that all the programs sampled for a tournament have a fitness rank between 1 and $j$ (i.e. are from $S_{1}, \ldots, S_{j}$ ) is given by

$$
\frac{\left(\begin{array}{c}
\sum_{i=1}^{j}\left|S_{i}\right| \\
k
\end{array}\right)}{\left(\begin{array}{c}
N \\
k
\end{array}\right)}
$$

Let $T_{j}$ be the event that the best ranked program in a tournament is from $S_{j}$. Therefore, the probability that the selected program will have rank $j$ is:

$$
P\left(T_{j}\right)=\frac{\left(\begin{array}{c}
\sum_{i=1}^{j}\left|S_{i}\right| \\
k
\end{array}\right)}{\left(\begin{array}{c}
N \\
k
\end{array}\right)}-\frac{\left(\begin{array}{c}
\sum_{i=1}^{j-1}\left|S_{i}\right| \\
k
\end{array}\right)}{\left(\begin{array}{c}
N \\
k
\end{array}\right)}
$$

Let $W_{j}$ be the event that the program $p \in S_{j}$ wins or is selected in a tournament. As each element of $S_{j}$ has equal probability of being selected in a tournament, the probability of the event $W_{j}$ is:

$$
P\left(W_{j}\right)=\frac{P\left(T_{j}\right)}{\left|S_{j}\right|}
$$

Therefore the probability that $p$ is selected at least once in $y$ tournaments is:

$$
P\left(E_{j, y}\right)=1-\left(1-P\left(W_{j}\right)\right)^{y}
$$

Replacing $P\left(W_{j}\right)$ we obtain Equation (11) as required.

For the simple situation that all individuals have distinct fitness values, $\left|S_{j}\right|$ becomes 1 . Therefore, by replacing and simplifying Equations (12) and (13), we obtain the following equation, which confirms the model presented in [4].

$$
P\left(W_{j}\right)=\frac{\left(\begin{array}{l}
j \\
k
\end{array}\right)-\left(\begin{array}{c}
j-1 \\
k
\end{array}\right)}{\left(\begin{array}{c}
N \\
k
\end{array}\right)}
$$

Following the main concern in the paper, the next section compares and analyses the selection behaviour in the two tournament selection schemes.

\section{SELECTION BEHAVIOUR COMPARISON AND ANALYSIS}

Inspired by [17], we split the loss of program diversity into two parts. One contribution is from the fraction of the population that are not sampled at all during the selection phase. The other contribution is from the fraction of population that never win any tournament. Based on the sampling probability models and the selection probability models (Equations 8 and 9 for standard tournament selection and Equations 10 and 11 for no-replacement tournament selection), we calculated the three loss of program diversity measures, namely total loss of program diversity and the contributions from not-sampled and not-selected individuals, for standard and no-replacement tournament selections on each of the four populations with different fitness rank distributions (see Figure 2).

When the tournament size is 1 , causing tournament selection be equivalent to random selection, the figure shows that total loss of program diversity is the result of the notsampled individuals only. This is because once an individual is sampled, it must be selected as a parent as there are no other competitors in the tournament. However, the contribution from not-sampled individuals reduces as the tournament size increases, while the contribution from not-selected individuals becomes larger and clearly dominates the total loss of program diversity when the tournament size is greater than five.

With all three of the loss of program diversity measures, there are no noticeable differences between the two tournament selection schemes or between the four populations with different FRDs (except one slight difference for the uniform FRD case). In all cases, the loss of program diversity is determined almost entirely by the tournament size.

Although it is difficult to prove the finding mathematically, the following brief analysis of the contribution from not-sampled individuals may help to explain the finding.

For standard tournament selection, according to Equation 8 , the probability that a program has never been sampled in $y=N$ tournaments is:

$$
\left(\left(\frac{N-1}{N}\right)^{N}\right)^{\frac{N}{N} k}=\left(\frac{N-1}{N}\right)^{N k} \approx e^{-k}
$$

for large $N$. The loss of program diversity contributed from not-sampled individuals can be calculated approximately by:

$$
\frac{1}{N} \sum_{i=1}^{N} e^{-k}=e^{-k}
$$

which is just a function of the tournament size $k$. Therefore, the trends of the loss of program diversity contributed from not-sampled individuals are almost the same in the 

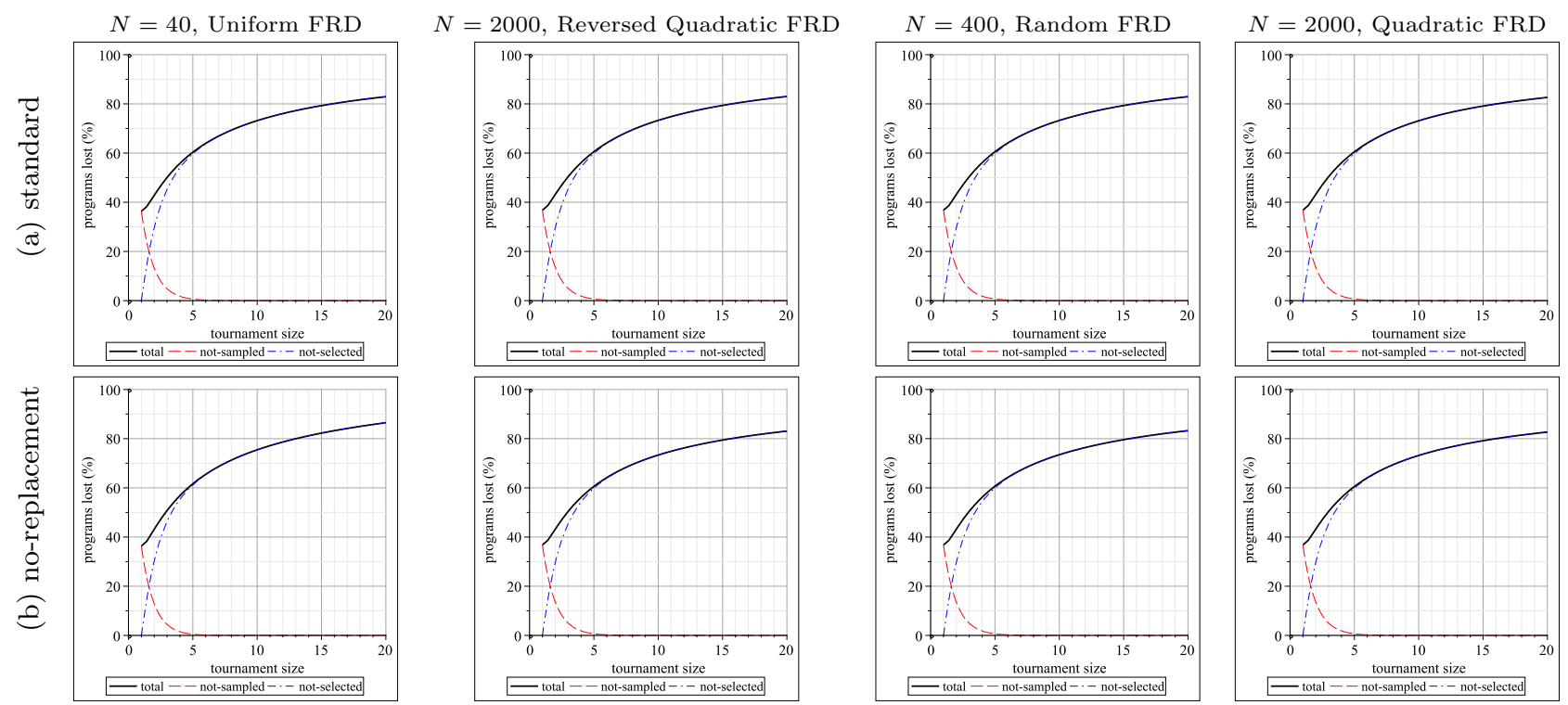

Figure 2: Loss of program diversity comparison. Note that the tournament size is discrete but the plots show curves to aid interpretation only.
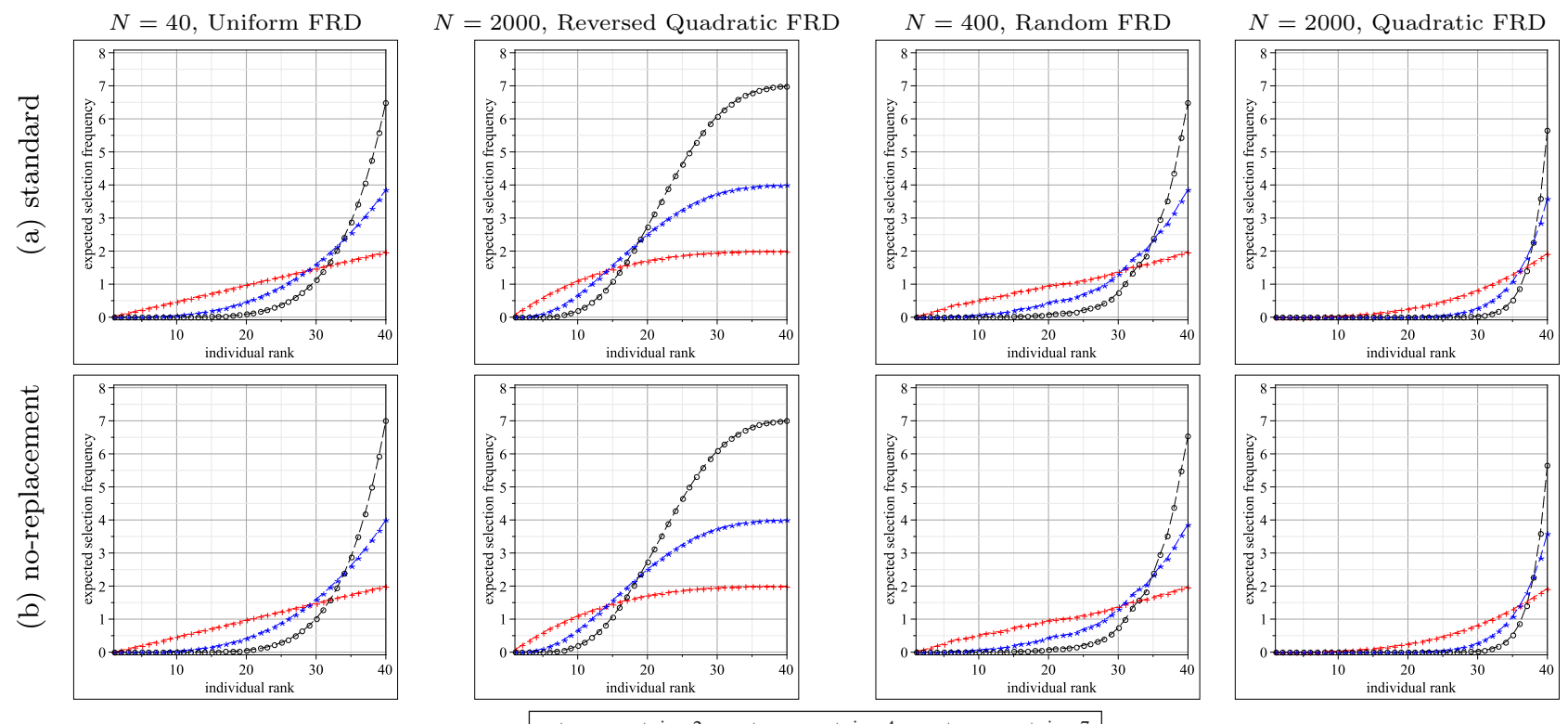

Figure 3: Selection frequency comparison.

four different sized populations with different fitness rank distributions. For the total loss of program diversity, we may obtain a function of a similar form after simplifying or approximating Equation 9.

For no-replacement tournament selection, according to Equation 10, the probability that a program has never been sampled in $y=N$ tournaments is:

$$
\left(\frac{N-k}{N}\right)^{N}=\left(\frac{\frac{N}{k}-1}{\frac{N}{k}}\right)^{\frac{N}{k} k} \approx e^{-k}
$$

for large $N / k$, which is approximately the same as that in standard tournament selection. This may explain why there is only a slight difference in no-replacement tournament selection on the smaller sized population when larger tournament sizes are used.
The simulations together with the above analyses imply that there is no noticeable difference between standard and no-replacement tournament selections in terms of the loss of program diversity when the size of a population is large.

Figure 3 shows the selection frequency for an individual at each rank for the two tournament selection schemes on the four populations with different FRDs.

Instead of plotting the expected selection frequency for each individual, we only plotted it for each of the 40 unique fitness ranks so that plots in different sized populations are in the same scale and it is easy to identify what fitness ranks may be lost. Further, we chose three different tournament sizes, namely 2,4 , and 7 as they are mostly commonly used in the literature, to illustrate how tournament size affects the selection behaviour. 

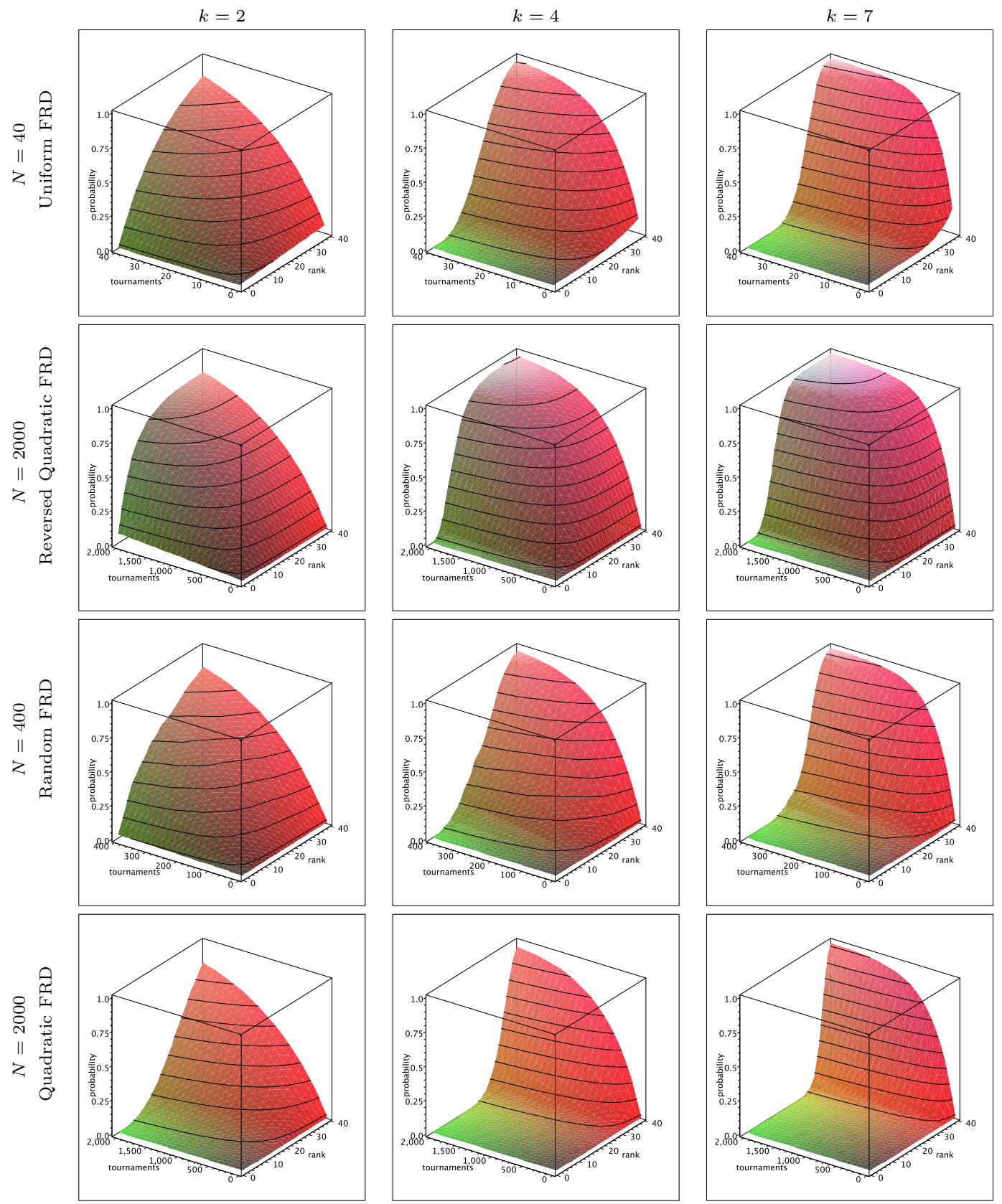

Figure 4: Selection probability distributions in no-replacement tournament selection scheme with tournament sizes 2,4 and 7 on four different fitness rank distributions. The tournament axis reflects the corresponding population size as the total number of tournaments required is the same as the population size (see Section 3).

The figure shows that both selection schemes favour better ranked individuals for all the tournament sizes. The selection pressure is more biased toward better ranked individuals as the tournament size increases. Overall, there is no noticeable difference between the corresponding selection frequencies in the two tournament selection schemes. A slight difference is observable in the leftmost graphs for the top ranked individuals when the tournament size is larger and the population size is smaller.

Additional visualisations of the loss of program diversity and the selection frequency on other sized populations with the four given fitness rank distributions support the findings consistently (figures are omitted due to the space limit).
We also calculated and plotted (see Figure 4) the selection probability distributions of the two tournament selection schemes using the three different tournament sizes on the four populations with different fitness rank distributions. Again, there is no noticeable difference between the corresponding selection probability distributions for standard and no-replacement tournament selection schemes (only noreplacement tournament selection scheme has been reported in Figure 4 due to the space limit).

All the results show that under the situation that common tournament sizes and population sizes are used, no significant difference in selection behaviour has been observed between the two tournament selection schemes. Therefore, 

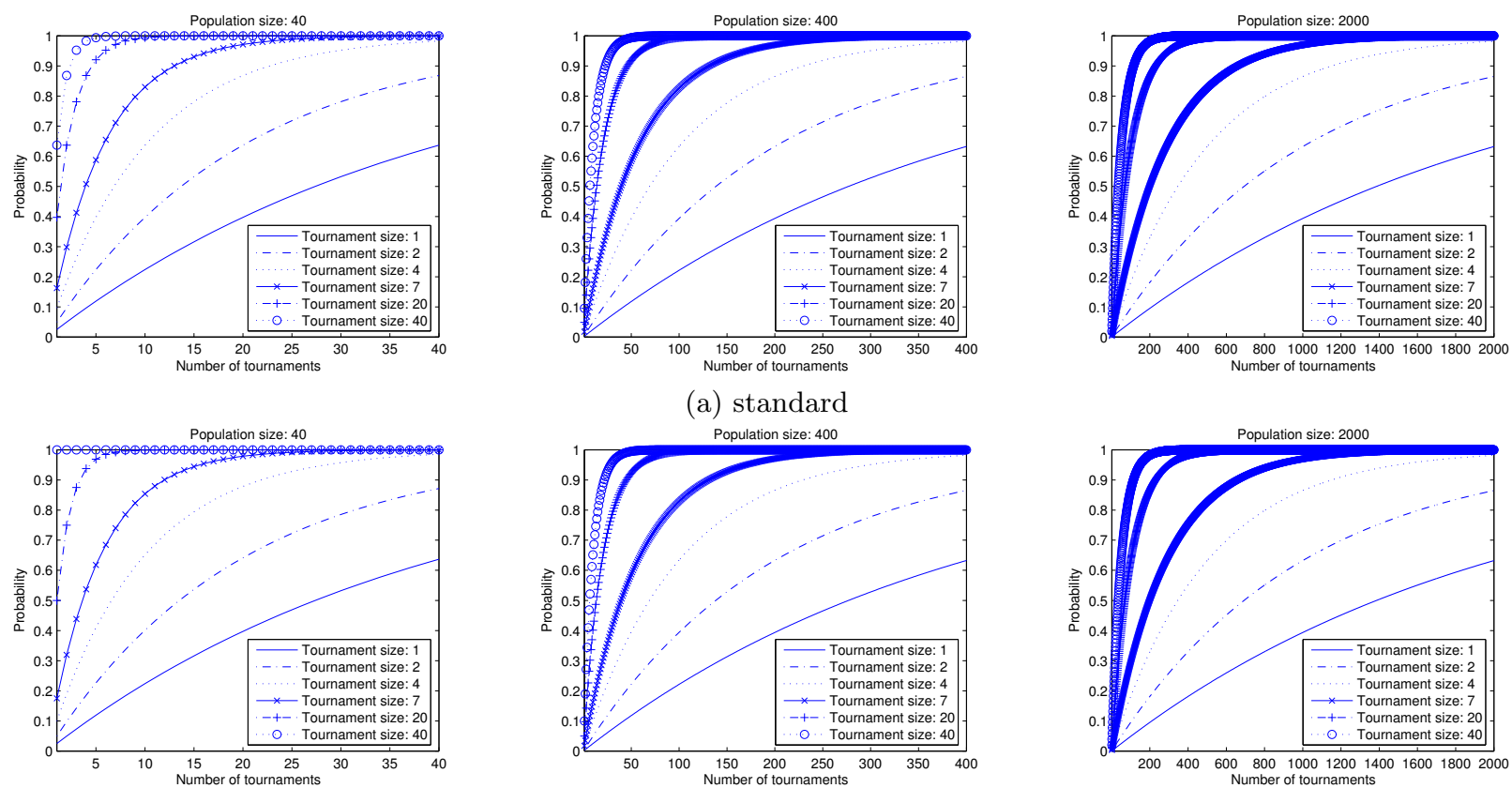

(b) no-replacement

Figure 5: Probability that a program is sampled at least once during the selection phase. (Note that the scales on the x-axes differ.)

the next section examines the sampling behaviour to explore the underlying reasons.

\section{SAMPLING BEHAVIOUR COMPARISON AND ANALYSIS}

Figure 5 shows the sampling behaviour in the two schemes via the probabilities of a program having been sampled using six tournament sizes in three populations of sizes 40, 400, and 2000 as the number of tournaments increases up to the corresponding population size.

Apart from the special case of population size 40 and tournament size 40 , which produces a $100 \%$ sampling probability in no-replacement tournament selection, there is no noticeable difference between corresponding schemes. The results are somehow surprising but are understandable since both Equations (8) and (10) can be simplified to $1-e^{-\frac{y}{N} k}$ for large $N$ and $N / k$.

\subsection{Significance in Similarity or Difference Analysis}

To further investigate the similarity or the difference between the sampling behaviour in the two tournament selection schemes, we ask the following question: for a given population of size $N$, if we keep sampling individuals with replacement, then after how many sampling events, will we have a certain level of confidence that there will be duplicates amongst the sampled individuals?

The answer is the minimum $k$ for which

$$
\frac{N !}{N^{k}(N-k) !}<\alpha
$$

where $N^{k}$ is the total number of different sampling results when sampling $k$ individuals with replacement, $\frac{N !}{(N-k) !}$ is the number of sampling events such that no duplicate is in the $k$ sampled individuals, and $(1-\alpha)$ is the confidence level.

Figure 6 illustrates the relationship between population size $N$, minimum tournament size $k$, and the confidence level $1-\alpha$. For instance, sampling individuals with replacement will sample duplicates in 7 sampled individuals with $99 \%$ confidence when the population size is about 2000, and $95 \%$ confidence when the population size is about 400 , but only $90 \%$ confidence when the population size is about 200 . We also calculated that when the population size is 40 , the confidence level is only about $57 \%$ for $k=7$. These results explained why we have only observed slight differences in the loss of program diversity and the selection frequency between the two schemes on the small population using larger tournament sizes.

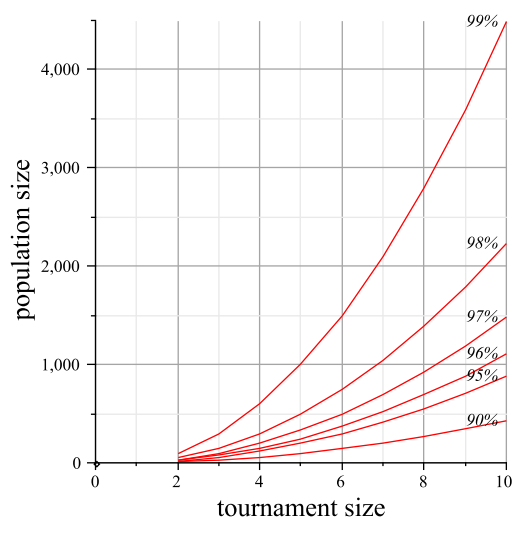

Figure 6: Confidence level, population size and minimum tournament size. Note that tournament size is discrete but the plot shows curves to aid interpretation only. 
The results show that for common tournament sizes 4 or less, we would not to expect to see any duplicates in anything except very small populations. Even for tournament size 7, we would expect to see some number of duplicates for populations less than 200. Therefore, for most common and reasonable settings of tournament sizes and population sizes, the multi-sampled issue seldom occurs in standard tournament selection. Eliminating the multi-sampled issue in standard tournament selection is very unlikely to significantly change the selection performance. Further, it is worth noting that duplicated individuals do not necessarily influence the result of a tournament when the duplicates have worse fitness values than other sampled individuals. Therefore, the probability of significant difference between standard tournament selection and no-replacement tournament selection will be even smaller. As a result the multi-sampled issue generally is not crucial to the selection behaviour in standard tournament selection.

Given the difficulty of implementing sampling-withoutreplacement in a parallel architecture, most researchers have abandoned the sampling-without-replacement, and used the simpler sampling-with-replacement scheme, hoping that the multi-sampled issue is not important. Our analysis results justified this choice.

\section{CONCLUSIONS}

Standard tournament selection samples individuals with replacement, which causes the multi-sampled issue. The multi-sampled issue can be overcame by no-replacement tournament selection. Through theoretical analyses and simulations of the two tournament selection schemes, the paper showed that when common tournament sizes and population sizes are used, the sampling-without-replacement strategy does not make the selection behaviour significantly different from that in standard tournament selection. The multisampled issue seldom occurs in standard tournament selection. Therefore, in general, the issue is not crucial to the selection behaviour in standard tournament selection.

This work was conducted with intention for GP, however the theoretical analyses are very general so that the results could be applied to any other EAs.

\section{Acknowledgment}

We would like to thank Simon Doherty and Kourosh Neshatian of School of Mathematics, Statistics and Computer Science at Victoria University of Wellington for their useful discussions, and the anonymous reviewers for valuable suggestions that helped to improve this paper.

\section{REFERENCES}

[1] T. Bäck. Selective pressure in evolutionary algorithms: A characterization of selection mechanisms. In Proceedings of the First IEEE Conference on Evolutionary Computation., pages 57-62, 1994.

[2] T. Blickle and L. Thiele. A mathematical analysis of tournament selection. In Proceedings of the Sixth International Conference on Genetic Algorithms, pages 9-16, 1995.

[3] T. Blickle and L. Thiele. A comparison of selection schemes used in evolutionary algorithms. Evolutionary Computation, 4(4):361-394, 1997.
[4] J. Branke, H. C. Andersen, and H. Schmeck. Global selection methods for SIMD computers. In Proceedings of the AISB96 Workshop on Evolutionary Computing, pages 6-17, 1996.

[5] A. Brindle. Genetic algorithms for function optimisation. PhD thesis, Deptartment of Computing Science, University of Alberta, 1981.

[6] M. Bulmer. The Mathematical Theory of Quantitative Genetics. Oxford University Press, Oxford, UK, 1980.

[7] V. Filipović, J. Kratica, D. Tošić, and I. Ljubić. Fine grained tournament selection for the simple plant location problem. In 5th Online World Conference on Soft Computing Methods in Industrial Applications, pages 152-158, 2000.

[8] D. E. Goldberg and K. Deb. A comparative analysis of selection schemes used in genetic algorithms. Foundations of Genetic Algorithms, pages 69-93, 1991.

[9] G. R. Harik. Finding multimodal solutions using restricted tournament selection. In Proceedings of the Sixth International Conference on Genetic Algorithms, pages 24-31, San Francisco, CA, 1995. Morgan Kaufmann.

[10] J. R. Koza. Genetic Programming - On the Programming of Computers by Means of Natural Selection. MIT Press, Cambridge, 1992.

[11] S. Luke and L. Panait. Fighting bloat with nonparametric parsimony pressure. In Proceedings of Parallel Problem Solving from Nature, volume 2439 of LNCS, pages 411-421. Springer, 2002.

[12] S. Luke and L. Panait. Lexicographic parsimony pressure. In Proceedings of the Genetic and Evolutionary Computation Conference, pages 829-836, 2002.

[13] B. L. Miller and D. E. Goldberg. Genetic algorithms, tournament selection, and the effects of noise. Technical Report 95006, University of Illinois at Urbana-Champaign, July 1995.

[14] B. L. Miller and D. E. Goldberg. Genetic algorithms, selection schemes, and the varying effects of noise. Evolutionary Computation, 4(2):113-131, 1996.

[15] T. Motoki. Calculating the expected loss of diversity of selection schemes. Evolutionary Computation, 10(4):397-422, 2002.

[16] H. Muhlenbein and D. Schlierkamp-Voosen. Predictive models for the breeder genetic algorithm, I: continuous parameter optimization. Evolutionary Computation, 1(1):25-49, 1993.

[17] R. Poli and W. B. Langdon. Backward-chaining evolutionary algorithms. Artificial Intelligence, 170(11):953-982, 2006.

[18] E. Popovici and K. D. Jong. Understanding EA dynamics via population fitness distributions. In Proceedings of the Genetic and Evolutionary Computation Conference 2003, pages 1604-1605, 2003.

[19] A. Sokolov and D. Whitley. Unbiased tournament selection. In Proceedings of GECCO 2005, pages 1131-1138. ACM Press, 2005.

[20] H. Xie, M. Zhang, and P. Andreae. Another investigation on tournament selection: modelling and visualisation. In Proceedings of GECCO 200\%, pages 1468-1475, 2007. 